\title{
Escorpionismo em Belo Horizonte, MG: um estudo retrospectivo
}

\author{
Scorpionism in Belo Horizonte, MG: a retrospective study
}

\author{
Marcely Regina Martins Soares ${ }^{1}$, Cristiano Schetini de Azevedo ${ }^{1}$ and Mário De Maria ${ }^{1}$
}

\begin{abstract}
Resumo Os escorpiões (Arachnida, Scorpiones), especialmente na área urbana, devido à grande densidade demográfica e possibilidade de confronto, representam risco à saúde pública. A espécie Tityus serrulatus é a mais importante, do ponto de vista médico, causando o maior número de acidentes. Objetivou-se levantar dados epidemiológicos e a ocorrência de acidentes escorpiônicos em Belo Horizonte, entre 1990 e 1997. Os dados foram obtidos no Hospital de Pronto Socorro João XXIII. Ocorreram 3265 acidentes, sendo a maioria em 1996. Aconteceram 6 acidentes fatais. Com maior incidência nos meses de janeiro, o sexo masculino, os membros superiores e a faixa etária entre 25 e 65 anos, foram os mais atingidos. Os resultados constituem importante subsídio para o controle do escorpionismo, pois delimitam as áreas mais atingidas e o perfil dos acidentados, permitindo campanhas educativas e de prevenção mais eficientes.
\end{abstract}

Palavras-chaves: Escorpionismo. Epidemiologia. Tityus serrulatus. Escorpião amarelo.

\begin{abstract}
Scorpions, especially in urban areas, due to the great demographic density and confrontation possibility, represent a risk to the public health. Tityus serrulatus is the most important species, causing the highest number of accidents. This study intended to raise epidemic data and the occurrence of scorpionism in Belo Horizonte, between 1990 and 1997. The data were gathered from the records of Hospital de Pronto Socorro João XXIII. Of the 3265 cases, most occurred in 1996, of which six were fatal accidents. With greater incidence in January, the male sex, superior members and 25-65 year-old age group were the most affected. The results constitute an important tool for the control of scorpionism, since they delimit the areas most involved and the victims' profile, enabling more efficient and durable prevention educational campaigns.
\end{abstract}

Key-words: Scorpionism. Epidemiology. Tityus serrulatus. Yellow scorpion.

Entre os problemas de saúde pública no Brasil, o escorpionismo desponta com peculiaridades próprias. Com maior destaque nas regiões climáticas mais quentes e recrudescimento nos meses onde ocorrem aumento da temperatura e da pluviosidade, os acidentes com escorpiões parecem ser bem mais freqüentes do que acusam as estatísticas oficiais, podendo assumir um perfil epidemiológico grave em determinadas áreas, sob certas circunstâncias ${ }^{14}$.

São relatados, no Brasil, casos de escorpionismo em quase todos os estados, principalmente em Minas Gerais e São Paulo, onde os escorpiões constituem problema de saúde pública. Os acidentes podem variar amplamente quanto à gravidade. Há casos de morte e de sequelas causadoras de incapacidade temporária para o trabalho e outras atividades habituais. A Família Buthidae é a maior e mais amplamente distribuída, não só no Brasil, mas em todo o mundo e, também, a mais importante do ponto de vista epidemiológico, incluindo as 25 espécies consideradas perigosas para o homem ${ }^{5}$.

No gênero Tityus Kock, 1836, as espécies representam $60 \%$ da fauna escorpiônica neotropical, podendo causar acidentes graves. É provável que todas as espécies deste gênero apresentem um veneno ativo sobre o homem, ainda que os principais acidentes se devam às espécies Tityus serrulatus Lutz \& Melo, 1922, Tityus bahiensis Perty, 1834, e Tityus stigmurus Thorell, $1877^{1518}$.

T. serrulatus ocorre na Bahia, Minas Gerais, Espírito Santo, Rio de Janeiro, São Paulo, Pará e Goiás. É de colorido amarelo claro, o que originou o seu nome popular: escorpião amarelo. Medem até $7 \mathrm{~cm}$ de comprimento. Suas populações são formadas apenas por fêmeas, cuja reprodução se dá por partenogênese ${ }^{511}{ }^{13}$. Alguns trabalhos consideram $T$. serrulatus componente de um complexo com T. stigmurus, apresentando quatro formas: confluenciata, confluenciata/maculata,

1. Instituto de Ciências Biológicas da Universidade Federal de Minas Gerais, Belo Horizonte, MG.

Endereço para correspondência: Dra Marcely Regina Martins Soares. Laboratório de Aracnologia/Deptº de Zoologia/ICB/UFMG. Av. Antônio Carlos 6627, Pampulha, 31270-907 Belo Horizonte, MG, Brasil.

Tel: 5531 3499-2916; Fax: $55313499-2899$.

E-mail: aracnologia ufmg@yahoo.com.br

Recebido para publicação em 4/9/2001. 
unifasciata e trifaciata. Duas delas (confluenciata/ maculata e trifaciata) se reproduzem sexualmente, ou seja, nesse complexo ( $T$. serrulatus/T. stigmurus), existiriam populações sexuais e partenogenética ${ }^{89}$, e não somente partenogenéticas. Cada fêmea de T. serrulatus pode ter três, quatro ou mais parições e cerca de 70 filhotes durante a vida ${ }^{11}$. Adaptam-se muito bem ao ambiente urbano e, quando encontram condições próprias, proliferam muito.

Em Belo Horizonte, cidade considerada como construída sobre solo escorpionífero ${ }^{2}$, a ocorrência de T. serrulatus e os acidentes por eles provocados representam uma situação preocupante. A ocorrência tem sido relacionada a fatores inerentes ao solo, como áreas de pedreiras, barrancos, marmorarias e também a construções antigas, serrarias e olarias ${ }^{1}$.

O objetivo deste trabalho é relatar a ocorrência de acidentes escorpiônicos em Belo Horizonte, Minas Gerais, no período de 1990 a 1997, relacionando-os com alguns dados epidemiológicos.

\section{MATERIAL E MÉTODOS}

Os dados referentes aos acidentes escorpiônicos, entre 1990 e 1997, foram obtidos junto ao Centro de Toxicologia do Hospital de Pronto Socorro João XXIII, único hospital que possui e administra o soro antiescorpiônico em Belo Horizonte. Foram analisadas fichas preenchidas na hora de entrada dos pacientes, contendo dados como sexo, idade e local da ferroada.

Os escorpiões capturados foram identificados por técnicos no Centro de Vigilância Sanitária e por médicos do HPS João XXIII, sempre que eram levados pelas vítimas.

\section{RESULTADOS}

A distribuição e a freqüência dos acidentes, por ano, estão indicados na Tabela 1. A distribuição e a frequência dos acidentes, por mês e estação (secafria e chuvosa-quente), estão indicados na Tabela 2.

Um total de 3265 acidentes escorpiônicos ocorreu em Belo Horizonte entre 1990 e 1997, em decorrência de ferroadas do escorpião amarelo $T$. serrulatus. A distribuição dos acidentes escorpiônicos, por faixa etária dos acidentados, está indicada na Tabela 3. A freqüência e distribuição dos acidentes, de acordo com o sexo dos acidentados, estão indicadas na Tabela 4. A Tabela 5 indica o local das ferroadas, mostrando sua distribuição anual e a freqüência de ocorrência.

\begin{tabular}{|c|c|c|}
\hline$\overline{A n o}$ & Freqüência & $\%$ \\
\hline 1990 & 191 & 5,9 \\
\hline 1991 & 362 & 11,2 \\
\hline 1992 & 394 & 12,2 \\
\hline 1993 & 387 & 12,0 \\
\hline 1994 & 396 & 12,3 \\
\hline 1995 & 521 & 16,1 \\
\hline 1996 & 544 & 16,8 \\
\hline 1997 & 437 & 13,5 \\
\hline Total & 3.232 & 100 \\
\hline
\end{tabular}

Acidentes em anos não-identificados: 33 (1\%)

Em 1991, ocorreu a morte de um indivíduo do sexo masculino, na faixa etária de 1-4 anos, e de um indivíduo do sexo feminino, na faixa etária de 5-14 anos. O ano onde ocorreu o maior número de mortes foi 1994, com
4 óbitos de indivíduos do sexo masculino, sendo 2 mortes na faixa etária de 5-14 anos, 1 morte na faixa etária de 25-34 anos e uma morte em faixa etária não identificada.

\section{DISCUSSÃO}

De 1990 a 1997 ocorreram 3.265 acidentes em todo o Município de Belo Horizonte. Todos os acidentes foram causados por escorpiões amarelos ( $T$. serrulatus). Esta espécie, embora primitivamente habitante do cerrado e de campos abertos, tornou-se bem adaptada à vida domiciliar urbana, possivelmente em decorrência da rápida e desorganizada colonização pelo homem das regiões originalmente ocupadas pelo aracnídeo. Além disso, esses animais adaptaram-se facilmente às condições oferecidas pelas moradias humanas, com grandes possibilidades de abrigos, como lixo, entulhos, pilhas de tijolos e telhas, e uma alimentação farta, com baratas e outros insetos ${ }^{37}$. A falta de competidores e de predadores, como macacos, quatis, seriemas, sapos e rãs, também permite a rápida proliferação de escorpiões, uma vez que esses dois fatores contribuem decisivamente para o controle populacional das espécies. 
Tabela 2- Distribuição dos acidentes escorpiônicos, por mês e estação do ano, em Belo Horizonte, entre 1990 e 1997.

\begin{tabular}{lrr}
\hline & \multicolumn{2}{c}{ Acidentes } \\
\cline { 2 - 3 } Mês & $\mathrm{N}^{\circ}$ & $\%$ \\
\hline Seca-fria & & \\
abril & 200 & 6,2 \\
maio & 198 & 6,1 \\
junho & 225 & 6,9 \\
julho & 258 & 7,9 \\
agosto & 282 & 8,7 \\
setembro & 298 & 9,2 \\
total & 1.461 & 45,0 \\
Chuvosa-quente & & \\
$\quad$ outubro & 296 & 9,1 \\
novembro & 312 & 9,6 \\
$\quad$ dezembro & 340 & 10,5 \\
janeiro & 349 & 10,7 \\
fevereiro & 234 & 7,2 \\
$\quad$ março & 256 & 7,9 \\
\hline Total & 1.787 & 55,0 \\
\hline Total geral & 3.248 & 100,0 \\
\hline Acidentes em meses não-identificados: $17(0,5 \%)$ &
\end{tabular}

Acidentes em meses não-identificados: $17(0,5 \%)$

Tabela 3 - Distribuição dos acidentes escorpiônicos, por faixa etária dos acidentados, em Belo Horizonte, entre 1990 e 1997.

\begin{tabular}{|c|c|c|c|c|c|c|c|c|c|c|}
\hline \multirow{2}{*}{$\begin{array}{l}\text { Faixa Etária } \\
\text { (em anos) }\end{array}$} & \multicolumn{9}{|c|}{ Ano } & \multirow[b]{2}{*}{$\%$} \\
\hline & 1990 & 1991 & 1992 & 1993 & 1994 & 1995 & 1996 & 1997 & Total & \\
\hline$<1$ & 0 & 0 & 1 & 1 & 0 & 1 & 1 & 0 & 4 & 0,1 \\
\hline $1-4$ & 7 & 16 & 18 & 15 & 12 & 23 & 28 & 14 & 133 & 4,2 \\
\hline $5-14$ & 35 & 67 & 62 & 61 & 62 & 78 & 75 & 59 & 501 & 15,7 \\
\hline $15-24$ & 50 & 73 & 98 & 81 & 84 & 130 & 104 & 90 & 714 & 22,4 \\
\hline $25-65$ & 88 & 192 & 201 & 219 & 221 & 250 & 279 & 242 & 1.707 & 53,4 \\
\hline$>65$ & 11 & 14 & 12 & 10 & 17 & 21 & 32 & 18 & 135 & 4,2 \\
\hline Total & 191 & 362 & 392 & 387 & 396 & 503 & 519 & 423 & 3.194 & 100,0 \\
\hline
\end{tabular}

Acidentes em faixas etárias não-identificadas: 71 (2,2\%)

Tabela 4 - Distribuição dos acidentes escorpiônicos, por sexo dos acidentados, em Belo Horizonte, entre 1990 e 1997.

\begin{tabular}{|c|c|c|c|c|c|c|c|c|c|c|}
\hline \multirow[b]{2}{*}{ Sexo } & \multicolumn{10}{|c|}{ Ano } \\
\hline & 1990 & 1991 & 1992 & 1993 & 1994 & 1995 & 1996 & 1997 & Total & $\%$ \\
\hline Feminino & 80 & 160 & 165 & 157 & 174 & 213 & 250 & 207 & 1.418 & 44,8 \\
\hline Masculino & 107 & 191 & 221 & 222 & 209 & 283 & 278 & 230 & 1.751 & 55,2 \\
\hline Total & 187 & 351 & 386 & 379 & 383 & 496 & 528 & 437 & 3.169 & 100,0 \\
\hline
\end{tabular}

Acidentes em sexos não-identificados: 96 (2,9\%)

Tabela 5 - Identificação do local da ferroada dos escorpiões, em Belo Horizonte, entre 1990 e 1997.

\begin{tabular}{lrrrrrrrrrr}
\hline & \multicolumn{10}{c}{ Ano } \\
\cline { 2 - 12 } Local da ferroada & 1990 & 1991 & 1992 & 1993 & 1994 & 1995 & 1996 & 1997 & Total & $\%$ \\
\hline Cabeça & 4 & 10 & 5 & 4 & 3 & 13 & 10 & 7 & 57 & 2,2 \\
Membro superior & 39 & 55 & 139 & 188 & 208 & 216 & 236 & 195 & 1.281 & 48,4 \\
Membro inferior & 68 & 132 & 136 & 143 & 126 & 124 & 182 & 145 & 1.062 & 40,1 \\
Múltipla & 1 & 5 & 2 & 4 & 4 & 8 & 3 & 2 & 29 & 1,1 \\
Tronco & 12 & 33 & 30 & 28 & 25 & 29 & 31 & 29 & 217 & 8,2 \\
\hline Total & 124 & 235 & 312 & 367 & 366 & 390 & 462 & 378 & 2.646 & 100,0 \\
\hline
\end{tabular}

Acidentes em locais não-identificados: 619 (19\%) 
O ano que apresentou a maior freqüência de ocorrências foi 1996, com 544 acidentes, representando $16,8 \%$ dos casos. Nesse ano, o efeito climático El niño provocou mais chuvas e aumento da umidade, condições necessárias para uma boa sobrevivência dos escorpiões. Logo em seguida, o ano de 1995, com 521 acidentes, o que correspondeu a 16,1\% dos casos. Em 1990, tivemos um menor número de acidentes, com 191 ocorrências, o correspondente a 5,9\% dos casos.

O maior número de acidentes ocorreu em janeiro, com $349(10,7 \%)$, seguido pelo mês de dezembro, com $340(10,5 \%)$ acidentes. Os meses de abril e maio apresentaram os menores números de casos (200 em abril e 198 em maio), contribuindo com 6,2\% e $6,1 \%$ dos casos, respectivamente. Os acidentes foram mais freqüentes nos meses quentes e chuvosos (55\%), no período de outubro a março, atingindo o seu pico em janeiro, do que nos meses secos e frios (45\%). Esses dados corroboram os encontrados por outros autores ${ }^{4} 610$.

De 1990 a 1997, a faixa etária que mais se acidentou foi a de 25 a 65 anos (faixa etária utilizada nas fichas preenchidas no Hospital João XXIII), com $1.707(53,4 \%)$ casos. Esse resultado pode ser devido à uma maior amplitude dessa faixa etária (40 anos) em relação às outras. A tentativa de se representar as diferentes fases de desenvolvimento do ser humano (recém nascido, bebê, criança, adolescente, adulto e idoso) talvez explique tal divisão. A faixa etária menos atingida foi a dos menores de 1 ano, com $4(0,1 \%)$ casos apenas. Nesse mesmo período, o maior número de acidentes ocorreu com pessoas do sexo masculino, perfazendo um total de $1.751(55,2 \%)$ casos, enquanto que indivíduos do sexo feminino foram registrados $1.418(44,8 \%)$ acidentes. Dados semelhantes foram encontrados num estudo de acidentes escorpiônicos na Grande São Paulo e Municípios próximos, entre os anos de 1989 e $1990^{17}$. Isso pode estar relacionado com o tipo de profissão exercida pelos homens. Estão mais sujeitos a serem ferroados os que trabalham em serrarias ou depósitos de madeira, os que lidam com tijolos em construções, os que trabalham com remoção de terra, olarias, pedreiras ou marmorarias ${ }^{2}$.

Foram registrados, durante o presente trabalho, $1.281(48,4 \%)$ acidentes atingindo os membros superiores, $1.062(40,1 \%)$ atingindo os membros inferiores, $57(2,2 \%)$ atingindo a região da cabeça e $29(1,1 \%)$ atingindo várias partes do corpo ao mesmo tempo - múltiplas. Os escorpiões são animais de hábitos noturnos, passando o dia escondidos em locais escuros, podendo ser confundidos com o ambiente ou parecer mortos, aumentando o risco de uma pessoa ser ferroada. O manuseio de materiais de construção ou entulhos, em residências ou em outros ambientes, aliado à limpeza doméstica provavelmente contribuiu para que esses resultados fossem encontrados. Estudos similares corroboram esses resultados ${ }^{1015}$

Ocorreram óbitos em 1991 e 1994, com predominância do sexo masculino e faixa etária de 514 anos. Adultos são vítimas mais freqüentes de ferroadas de escorpiões, mas a morbidade é maior entre as crianças e idosos ${ }^{16}$. Sabe-se que vários fatores influenciam na gravidade dos acidentes causados por escorpiões, dentre eles, a espécie, o tamanho do escorpião, a quantidade de peçonha inoculada, a região do corpo atingida, a idade e sensibilidade da vítima ao veneno do animal. Crianças abaixo de sete anos e pessoas idosas constituem grupo de risco, uma vez que o sistema imune está em formação ou debilitado. Mas adultos sadios não estão imunes a ferroadas de escorpiões, sendo conhecidos muitos casos fatais ${ }^{12}$.

De 1990 a 1997, ocorreram óbitos em 0,2\% dos casos de acidentes escorpiônicos. Um estudo realizado no início da década de 40, apurou 145 óbitos em 2.449 acidentes (5,9\% dos casos) causados por $T$. serrulatus, em Belo Horizonte ${ }^{10}$. Esses dados mostram que, apesar do grande número de acidentes, as mortes têm sido raras e que os tratamentos médicos, quando necessários, têm sido eficazes.

Os resultados obtidos constituem importante ferramenta no subsídio à campanhas educativas, com vistas ao controle preventivo desses animais. Em Belo Horizonte, a ação simultânea no controle de locais onde há aparecimento de escorpiões é realizada em algumas etapas: captura, mapeamento e campanha educativa da população, numa ação preventiva. Para isso, é distribuída uma cartilha contendo orientações sobre cuidados que devem ser tomados dentro e fora do imóvel para prevenir o aparecimento desses artrópodes. Este controle deve ser realizado ao longo do ano, devendo ser mais intenso nos períodos que antecedem, ou mesmo durante, as épocas mais chuvosas.

O problema é basicamente sócio-cultural e econômico, e os programas de controle, propostos pelo Departamento de Controle de Zoonoses de Belo Horizonte, deverão ser aplicados continuamente, ano após ano, e só apresentarão resultados positivos quando a população apresentar mudanças comportamentais que se refletirão na diminuição dos índices de acidentes e infestação. Para isso, poderiam ser realizadas palestras em escolas, visando o esclarecimento e a conscientização das crianças e principalmente dos professores sobre acidentes, biologia dos escorpiões, e de como proceder em caso de ferroadas, e também, palestras para profissionais, com o intuito de ampliar os conhecimentos na identificação correta dos espécimes capturados. 


\section{AGRADECIMENTOS}

Ao Grupo de Vigilância Epidemiológica da Secretaria Municipal de Saúde de Belo Horizonte, ao Departamento de Controle de Zoonoses do Distrito Sanitário de Venda Nova, especialmente à Francisco José Reis dos Santos, e ao Centro de Toxicologia do Hospital de Pronto Socorro João XXIII, pelo fornecimento dos dados. Ao Professor Dr. Pedro Marcos Linardi, pelas sugestões dadas ao manuscrito.

\section{REFERÊNCIAS BIBLIOGRÁFICAS}

1. Bucherl W. Escorpiões e escorpionismo no Brasil. Memórias do Instituto Butantan 29: 243-253, 1959.

2. Bucherl W. Escorpionismo no Brasil. Memórias do Instituto Butantan 34: 9-24, 1969.

3. Bucherl W. Acúleos que matam. Revista dos Tribunais, São Paulo, 1979.

4. Campos JA, Silva OS, Lopez M, Freire-Maia L. Signs, symptoms and treatment of severe scorpion poisoning in children. In: Eaker D, Wadströn T (eds) Natural Toxins, Pergamon, New York, p. 61 68. 1980.

5. Candido DM. Escorpiões. In: Joly CA, Bicudo CEM (eds) Biodiversidade do Estado de São Paulo, Fundação de Amparo à Pesquisa do Estado de São Paulo, São Paulo, p. 25-34, 1999.

6. Eickstedt VRD. Escorpionismo por Tityus stigmurus no nordeste do Brasil (Scorpiones: Buthidae). Memórias do Instituto Butantan 47/48: 133-137, 1983.

7. Likes K, Banner Jr W, Chavez M. Centruroides exilicauda envenomation in Arizona. Western Journal of Medicine 141: 634647, 1984 .

8. Lourenço WR. Sur la systématique des scorpions appartenant au "complexe" Tityus stigmurus (Thorell, 1877) (Buthidae). Revista Brasileira de Biologia 41: 351-362, 1981.

9. Lourenço WR, Cloudsley-Thompson JL. Discovery of a sexual population of Tityus serrulatus, one of the morphs within the complex Tityus stigmurus (Scorpiones, Buthidae). The Journal of Arachnology 27: 154-158, 1999.
10. Magalhães $O$, Guimarães R. Algumas observações sobre acidentes pela picada de escorpiões. Brasil Médico 55: 466-467, 1941.

11. Matthiensen FA. The breeding of T. serrulatus Lutz \& Mello, 1927 in captivity (Scorpiones, Buthidae). Revista Brasileira de Pesquisas Médicas e Biológicas 4: 299-300, 1971.

12. Matthiensen FA. Os escorpiões e suas relações com o homem: uma revisão. Ciência e Cultura 40: 1168-1172, 1988.

13. Mello-Leitão C. Revisão do Gênero Tityus. Physiology 17: 57-76, 1939.

14. Neto CC, Filho PG, Yassuda CRW. Ensaio de campo no controle de escorpiões ( $T$. serrulatus) empregando Diazinon microencapsulado, na cidade de Aparecida, SP. Revista da Sociedade Brasileira de Medicina Tropical 27: 123, 1994.

15. Pessôa SB. Nota sobre alguns escorpiões do Gênero Tityus e Bothriurus. Anais Paulistas de Medicina e Cirurgia 29: 429-436, 1935.

16. Polis GA. The biology of scorpions. Stanford University Press, Stanford, 1980

17. Ribeiro LA, Navarro ALC, Valadares ES, Souza FG, Vieira GV, Souza GG, Cunha JR, Jorge MT. Epidemiologia do acidente por escorpião na Grande São Paulo e Municípios próximos, HVB, 1989/90. Revista da Sociedade Brasileira de Medicina Tropical 29: 238, 1996.

18. Schvarstman S. Plantas venenosas e animais peçonhentos. Editora Sarvier, São Paulo, 1992. 